\title{
PREVISÃO DE DESEMPENHO DE MOINHO VERTICAL DE LABORATÓRIO USANDO UM MOINHO DE BOND
}

\author{
D. C. ROCHA ${ }^{1 *}$, D. B. MAZZINGHY ${ }^{2}$, C. A. PEREIRA ${ }^{3}$ \\ Colorado School of Mines, Mining Department, Golden, Colorado, United States ${ }^{1}$ \\ Universidade Federal de Minas Gerais, Departamento de Minas, Belo Horizonte, Minas Gerais, Brasil ${ }^{2}$ \\ Universidade Federal de Ouro Preto, Departamento de Minas, Ouro Preto, Minas Gerais, Brasil ${ }^{3}$ \\ dnlcrocha@gmail.com*
}

Submetido 13/11/2017 - Aceito 13/11/2017

DOI: $10.15628 /$ holos.2017.6385

\section{RESUMO}

Os moinhos de bolas são muito ineficientes em aplicações de moagem fina, por isso, os moinhos verticais, reconhecidamente mais eficientes que os moinhos de bolas, vêm se tornando cada vez mais populares na indústria mineral. Esse trabalho apresenta uma proposta de uma nova metodologia para previsão de distribuição granulométrica de um moinho vertical à partir do uso de um moinho de Bond. O Modelo do Balanço Populacional (PBM) foi utilizado para descrever um moinho vertical de laboratório e um moinho de
Bond. Os parâmetros de quebra obtidos foram comparados com o intuito de se analisar a possibilidade de previsão de desempenho de um moinho vertical utilizando um moinho de Bond. A grande vantagem desse método é que a maioria dos laboratórios de processamento mineral já possuem um moinho de Bond para a realização dos testes. Resultados preliminares mostram a possibilidade de prever o desempenho de um moinho vertical de laboratório usando um moinho de Bond.

PALAVRAS-CHAVE: Moinho vertical, modelo do balanço populacional, moinho de bond, simulação, parâmetros de quebra.

\section{PERFORMANCE PREDICTION OF A LABORATORY VERTICAL STIRRED MILL USING A BOND BALL MILL}

\begin{abstract}
The use of ball mills for grinding in fine size ranges is inefficient and, therefore, vertical stirred grinding mills are becoming increasingly popular in the mineral processing industry due to its already known high energy efficiency. This work presents a hypothesis of a new methodology to predict the product size distribution of a vertical stirred mill using a Bond ball mill. The Population Balance Model (PBM) was used to empirically analyze the performance of a vertical mill and a Bond ball mill. The breakage parameters obtained
\end{abstract}

for both grinding mills are compared to determine the possibility of predicting the product size distribution of a vertical mill based on the results obtained from the Bond ball mill. The biggest advantage of this methodology is that most of the minerals processing laboratories already have a Bond ball mill to perform the tests suggested in this study. Preliminary results show the possibility of predicting the performance of a laboratory vertical stirred mill using a Bond ball mill.

KEYWORDS: Vertical stirred mill, population balance model, Bond ball mill, simulation, breakage parameters. 


\section{INTRODUÇÃO}

Moinhos verticais de carga agitada vêm sendo utilizados de forma eficiente no processo de moagem fina de minérios. O primeiro moinho vertical, chamado Tower Mill, foi desenvolvido no Japão pela Kubota Tower Mill Corporation, KTM (Stief, et al., 1987). Atualmente o Tower Mill é produzido pela Nippon-Eirich (Eirich, 2014). O moinho Vertimill tem o mesmo princípio de funcionamento e é fabricado pela Metso. O moinho vertical consiste em um corpo vertical, estacionário, um agitador de dupla hélice de baixa velocidade, um classificador externo e uma bomba de reciclagem. Ambos são moinhos induzidos por gravidade que usam carga moedora de alta densidade para promover a quebra do material. A ação de rotação e de elevação das partículas dentro do moinho gerada pelo agitador helicoidal é responsável pelo movimento dos corpos moedores e pelo mecanismo de quebra promovido pelo moinho sobre o material (Stief, et al., 1987). A Figura 1 mostra os principais componentes do Tower Mill e do Vertimill.

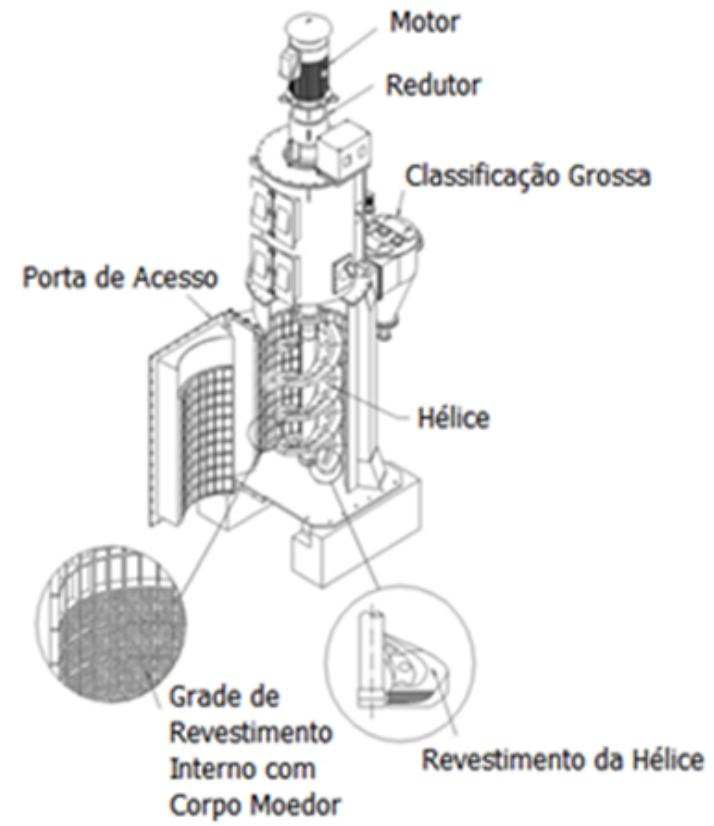

(a)

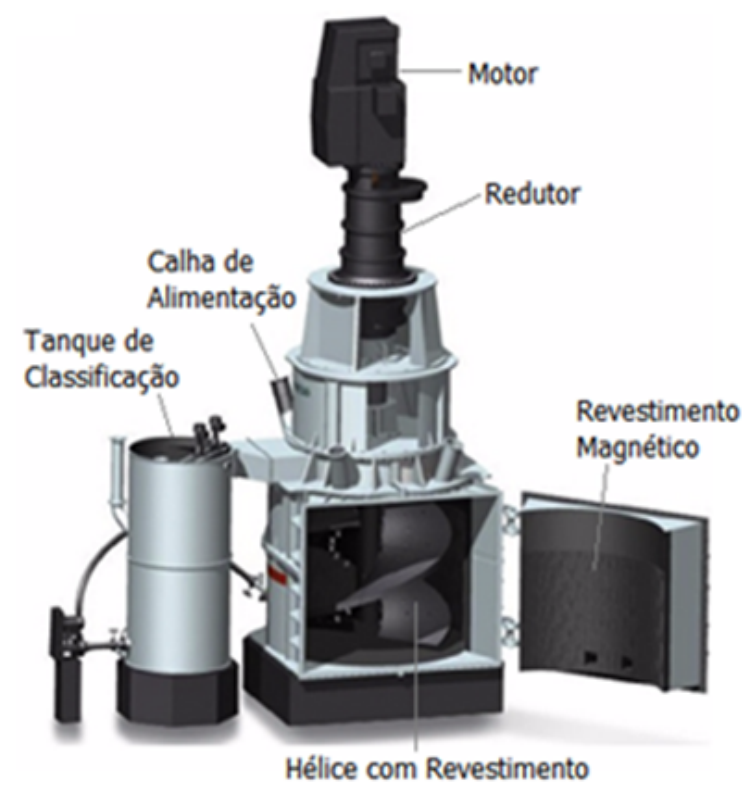

(b)

Figura 1: esquema do moinho (a) Tower Mill (cortesia Eirich) e do moinho (b) Vertimill (cortesia Metso).

Os moinhos verticais utilizam carga moedora de menor diâmetro quando comparado ao moinho de bolas. A maior eficiência energética de moinhos verticais está, entre outros fatores, relacionada ao menor tamanho de corpos moedores utilizados, o que aumenta a área superficial total disponível para a quebra do minério. Outros fatores que influenciam a maior eficiência energética desses moinhos são: (a) alta probabilidade de uma partícula de minério ser capturada entre dois corpos moedores para sua quebra, (b) maior frequência de colisões entre os corpos moedores, e (c) o equipamento possui um menor número de partes móveis se comparado ao moinho de bolas (Schonert, 1990).

O moinho de Bond consiste em um moinho tubular de bolas, sem levantadores de carga, de tamanho $305 \mathrm{~mm}$ de diâmetro por $305 \mathrm{~mm}$ de comprimento. O teste de Bond é usado na determinação do índice de trabalho de Bond, utilizado para calcular a energia necessária para se 
reduzir um material de, teoricamente, um tamanho infinito até $80 \%$ passante em $100 \mu \mathrm{m}$ (Wills \& Napier-Munn, 2006).

O objetivo desse estudo é analisar a possibilidade de previsão de desempenho de um moinho vertical de laboratório usando um moinho de Bond. As condições operacionais para realização de um teste de Bond são utilizadas nesse estudo devido ao vasto entendimento dessa metodologia e fácil acesso a moinhos de Bond em laboratórios de processamento mineral.

\subsection{Modelo do Balanço Populacional}

Em processos de cominuição, o modelo do balanço populacional é utilizado para estudar a evolução da redução de tamanho de partículas. O comportamento de quebra de cada grupo ou fração de partículas em um determinado tamanho pode ser calculado pelo uso desse modelo.

A Equação 1 descreve o aparecimento e desaparecimento das partículas em um moinho através do modelo do balanço populacional. Essa equação permite o cálculo da distribuição final de tamanho de partículas após submetidas a um processo de cominuição em função do tempo para processos em batelada (Austin, et al., 1984).

$\frac{d m_{i}(t)}{d t}=-S_{i} m_{i}(t)+\sum_{j=1}^{i-1} b_{i j} S_{j} m_{j}(t), \quad i=1,2, \ldots n$

Sendo $m_{i}(t)$ a massa final de partículas contidas no intervalo de tamanho $i$ após um tempo de moagem $t ; S_{i}$ a função seleção para as partículas contidas no intervalo de tamanho $i$; e $b_{i j}$ a função quebra de partículas contidas no intervalo de tamanho $i$ produzidas pela quebra de partículas no intervalo $j$.

A função seleção representa a velocidade de quebra de partículas em um determinado intervalo de tamanho. Essa velocidade pode ser calculada através da Equação 2, assumindo que a massa total de partículas dentro do moinho sela constante e que o valor da função seleção seja em função do tempo de moagem (Austin, et al., 1984).

$m_{i}(t)=m_{i}(0) e^{\left(-S_{i} t\right)}$

Sendo $m_{i}(t)$ a massa final de partículas contidas no intervalo de tamanho $i$ após um tempo de moagem $t ; m_{i}(0)$ a massa inicial de partículas no intervalo de tamanho $i$; e $S_{i}$ a função seleção para as partículas contidas no intervalo $i$.

A função quebra acumulada pode ser calculada de uma forma simples através do Método II desenvolvido por Austin et al. (1984), conforme mostrado na Equação 3.

$B_{i, j}=\frac{\log \left(\frac{1-P_{i}(0)}{1-P_{i}(t)}\right)}{\log \left(\frac{1-P_{2}(0)}{1-P_{2}(t)}\right)}$

Sendo $B_{i, j}$ a função quebra acumulada de partículas contidas no intervalo de tamanho $i$ produzidas pela quebra de partículas no intervalo $j ; P_{i}(0)$ a massa inicial de partículas acumuladas menor que o tamanho $i$; e $P_{i}(t)$, massa de partículas acumuladas menor que o tamanho $i$ após um tempo de moagem $t$. A função quebra em sua forma não-acumulada $b_{i, j}$ é calculada conforme mostrado na Equação 4 (Austin, et al., 1984):

$b_{i, j}=B_{i, j}-B_{i+1, j}$ 


\section{METODOLOGIA}

\subsection{Amostras}

Uma amostra de granito utilizado para pavimentação e construção foi utilizada nos testes em laboratório. $\mathrm{O}$ tamanho inicial das partículas era de aproximadamente $20 \mathrm{~mm}$ de diâmetro. A amostra foi inicialmente cominuída usando um britador de mandíbulas e o produto foi posteriormente reduzido utilizando-se um britador de rolos em duas etapas. O produto final foi classificado por tamanho e cada intervalo de tamanho separado em amostras individuais. A Tabela 1 identifica os cinco intervalos de tamanhos utilizados nos testes de moagem.

Tabela 1: Intervalos de tamanhos de partículas utilizados nos testes de moagem

\begin{tabular}{cc}
\hline Intervalo & Intervalo de Tamanho $(\boldsymbol{\mu m})$ \\
\hline $\mathbf{1}$ & $-841+595$ \\
$\mathbf{2}$ & $-595+420$ \\
$\mathbf{3}$ & $-420+297$ \\
$\mathbf{4}$ & $-297+210$ \\
$\mathbf{5}$ & $-210+149$ \\
\hline
\end{tabular}

\subsection{Moinho de Bond}

Um moinho de Bond foi utilizado para coletar os parâmetros de quebra, funções quebra e seleção, a serem usados no modelo do balanço populacional. O método Bll anteriormente descrito foi utilizado para calcular a função quebra acumulada. Utilizou-se 1890 gramas de material para cada um dos cinco diferentes intervalos de tamanho testados e tempo de moagem igual a 20 segundos. A carga de bolas de Bond para um teste de Índice de Trabalho foi utilizada nos testes. A velocidade de rotação do moinho foi mantida constante a $70 \mathrm{rpm}$ para todos os testes, e a moagem foi executada a seco, conforme o teste padrão de Bond para determinação do Índice de Trabalho. Para o cálculo da função seleção foram selecionados seis intervalos de tempos de moagem para obtenção da distribuição de tamanho do produto após cada intervalo de tempo, são eles: 20, 55, 130, 195, 285 e 405 segundos.

\subsection{Moinho vertical de carga agitada}

Para o presente estudo foi fabricado um moinho vertical de laboratório em escala de 1/20 em relação a geometria do moinho industrial Metso Vertimill VTM-1500. Um variador de velocidade do agitador foi instalado para operar na faixa de 50 à $400 \mathrm{rpm}$. A Figura 2 mostra o moinho vertical utilizado nos testes.

A Tabela 2 apresenta as condições operacionais utilizadas nos testes em moinho vertical para coleta dos parâmetros de quebra. Para o cálculo da função quebra, o tempo de moagem selecionado foi de 30 segundos. Para o cálculo da função seleção foram selecionados quatro intervalos de tempos de moagem para obtenção da distribuição de tamanho do produto após cada intervalo de tempo, são eles: 30, 85, 155 e 275 segundos. 
Tabela 2: Condições operacionais utilizadas nos testes com moinho vertical

\begin{tabular}{lll}
\hline Corpo moedor & $\mathrm{mm}$ & 9.5 \\
\hline Velocidade do Agitador & $\mathrm{rpm}$ & 130 \\
\hline Densidade do corpo moedor & $\mathrm{kg} / \mathrm{m}^{3}$ & 7850 \\
\hline Massa total de corpo moedor & $\mathrm{kg}$ & 20.2 \\
\hline Densidade do minério & $\mathrm{kg} / \mathrm{m}^{3}$ & 2700 \\
\hline Massa de minério & $\mathrm{kg}$ & 1.615 \\
\hline Porcentagem de Sólidos em massa & $\%$ & 35 \\
\hline
\end{tabular}

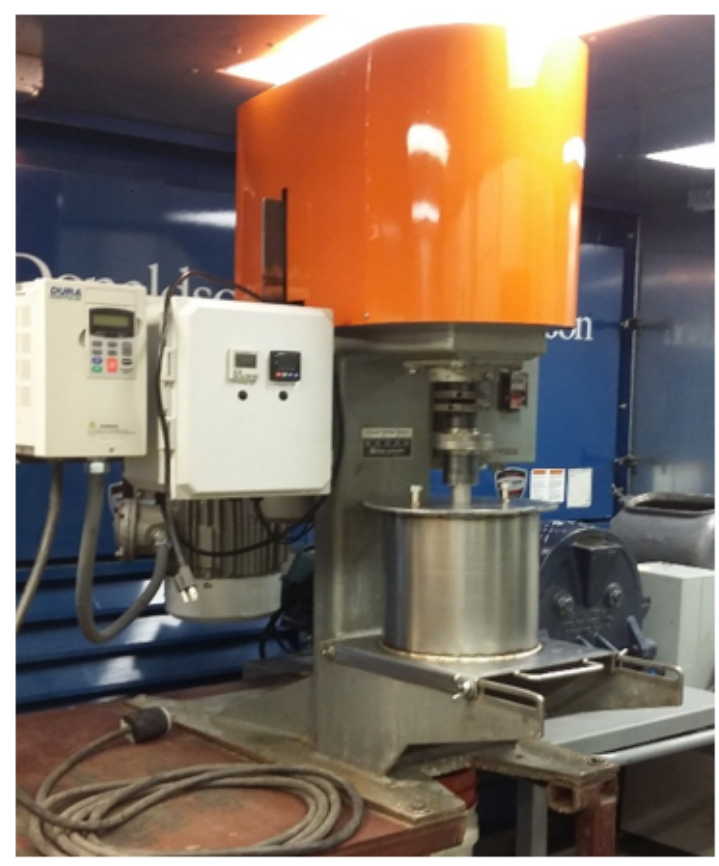

(a)

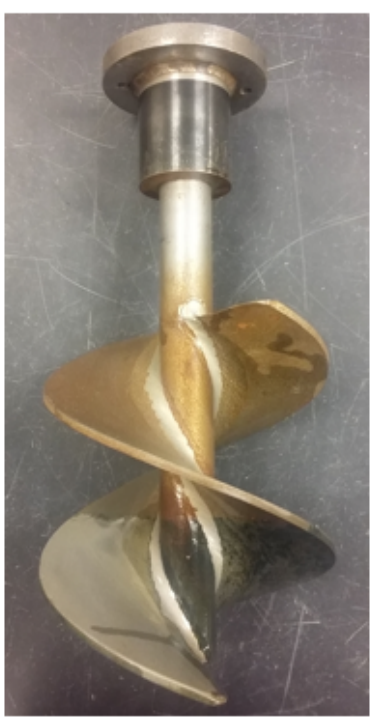

(b)

Figura 2: (a) moinho vertical de dupla hélice em escala laboratorial e (b) agitador.

\section{RESULTADOS E DISCUSSÃO}

\subsection{Determinação dos Parâmetros de Quebra}

Os parâmetros de quebra, funções quebra e seleção, foram determinados à partir de testes físicos em laboratório. A metodologia definida por Austin et al. (1984) foi utilizada nesse estudo. A Figura 3 mostra a função quebra determinada para os moinhos de Bond e para o moinho vertical.

A função quebra representa uma distribuição de tamanhos $i$ gerados à partir da quebra de uma partícula de tamanho $j$. Esse parâmetro é calculado à partir das massas de partículas geradas em cada intervalo de tamanho $i$ com relação à massa da partícula $j$ que foi submetida à quebra. $O$ processo de cominuição em um moinho de bolas ocorre através da combinação de atrito entre partículas e, principalmente, através de impacto dos corpos moedores sobre as partículas. A distribuição de tamanhos de um produto de moagem é diretamente relacionada aos eventos de quebra e ao tipo de material que está sendo cominuído. O centro de pesquisa mineral Julius 
Kruttschnitt (JKMRC) afirma que a função quebra é dependente do tipo de material a ser cominuído e da energia de quebra promovida pelo equipamento sobre o material (Morrel, et al., 1993). Se a energia de quebra promovida pelo equipamento for menor que a energia necessária para fraturar a partícula, essa não irá se quebrar e poderá ser somente afetada com fraturas internas. Mas se a energia de quebra for maior que a necessária para promover a geração de fraturas na partícula e sua posterior quebra, esse excesso de energia poderá ser usado em quebras secundárias sobre os fragmentos primários gerados.

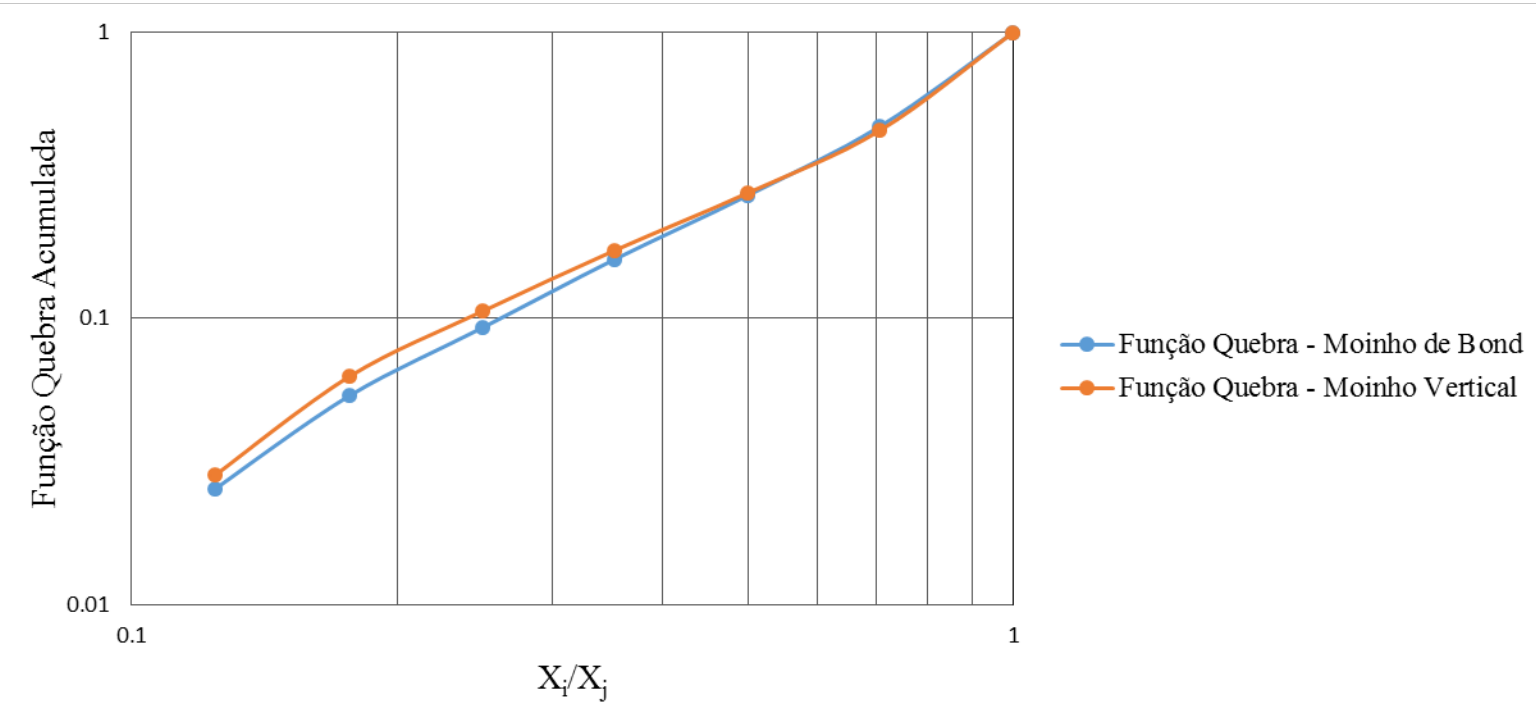

Figura 3: Funções quebra determinadas utilizando um moinho de Bond e um moinho Vertical.

As funções quebra obtidas para o moinho de Bond e moinho vertical mostram similaridade para o intervalo de tamanho número 1 analisado. As diferenças existentes podem ser provenientes do tipo de energia de quebra promovida pelo equipamento sobre as partículas. Moinhos verticais de carga agitada apresentam uma maior frequência de colisões de baixa energia e uma menor frequência de colisões de alta energia se comparado aos moinhos de bolas (Morrison, et al., 2009).

A Figura 4 mostra as funções seleções obtidas para os moinhos de Bond e vertical em função da fração granulométrica e o ajuste realizado. É possível interpretar que a velocidade de quebra das partículas mais finas é maior no moinho vertical que no moinho de Bond testados. $O$ tamanho dos corpos moedores é a variável que mais influencia a moagem, portanto, a maior taxa de quebra obtida no moinho vertical para as partículas finas pode estar relacionada com o tamanho das bolas utilizadas $(9,5 \mathrm{~mm})$. O comportamento linear das curvas de função seleção para os dois moinhos testados indica uma relação de boa proporcionalidade entre o tamanho máximo de bolas usado e o tamanho de partícula testado. 


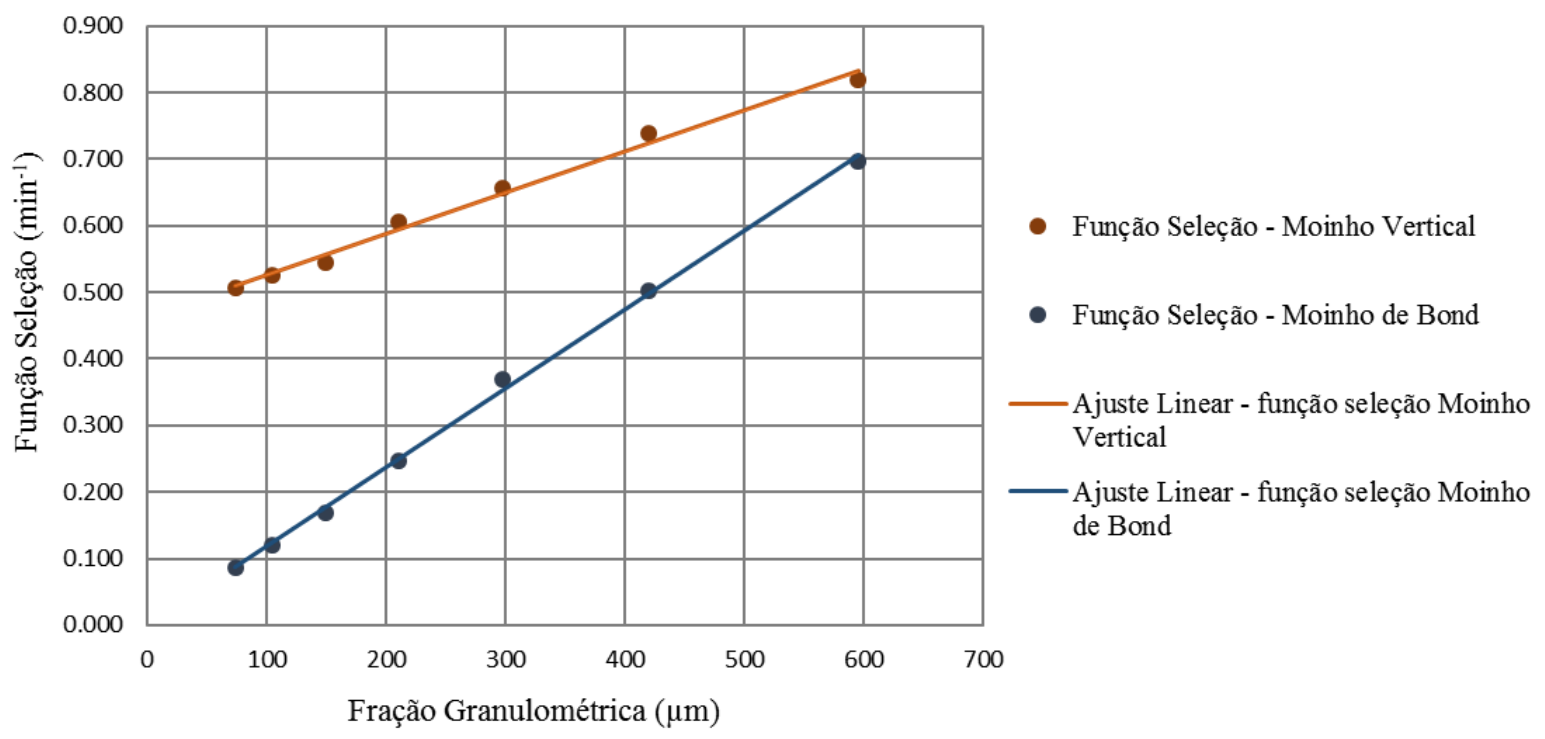

Figura 4: Funções seleções obtidas para os moinhos vertical e de Bond.

A Figura 5 mostra que existe uma relação entre os valores da função seleção obtidos para o moinho de Bond e os valores obtidos para o moinho vertical.

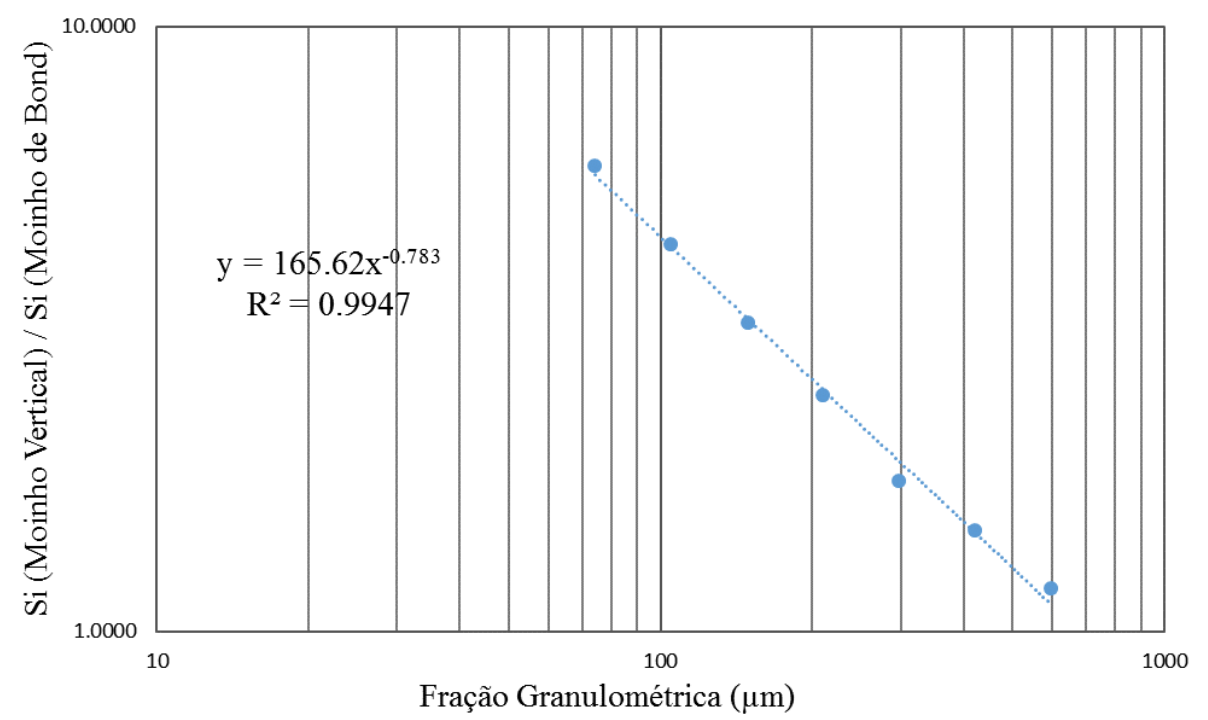

Figura 5: Relação entre funções seleção obtidas em moinho de Bond e moinho vertical.

A relação de potência encontrada entre as duas funções seleção medidas indica que os valores aumentam a uma taxa específica. $O$ valor de R-quadrado de 0.9947 sugere que um ajuste quase perfeito existe entre os dados. À partir da Equação 5, obtida pela curva do gráfico da figura 5 , é possível calcular a função seleção para o moinho Vertical.

$S i_{M V}=S i_{M B} 165.62 p^{-0.783}$

Onde $S i_{M V}$ representa a função seleção para o moinho vertical; $S i_{M B}$, a função seleção para o moinho de Bond; e $p$ o tamanho da faixa granulométrica em $\mu \mathrm{m}$.

\subsection{Previsão de Distribuição de Tamanho do Produto}

As Figuras 6 e 7 mostram a distribuição de tamanhos de produto medidas nos moinhos de Bond e vertical, respectivamente, assim como o resultado obtido pela simulação utilizando os 
parâmetros de quebra obtidos para quatro diferentes intervalos de tempo de moagem e alimentação na faixa de tamanho $-841+595 \mu \mathrm{m}$. Os parâmetros de quebra determinados para a amostra de granito são capazes de estimar com acurácia a distribuição final de produtos em função do tempo de moagem para os dois moinhos testados.

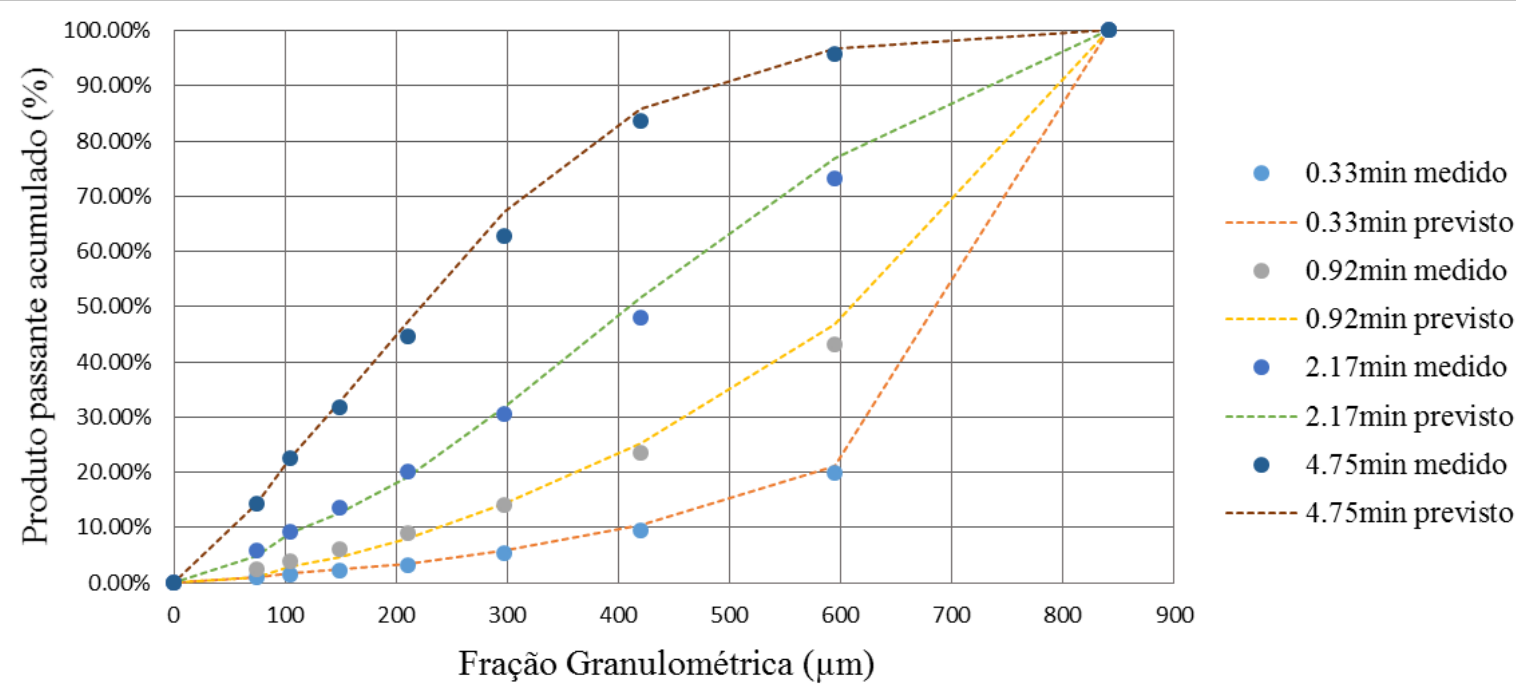

Figura 6: Previsão de produto final de um moinho de Bond em função do tempo de moagem (linha pontilhada) e os valores medidos (pontos).

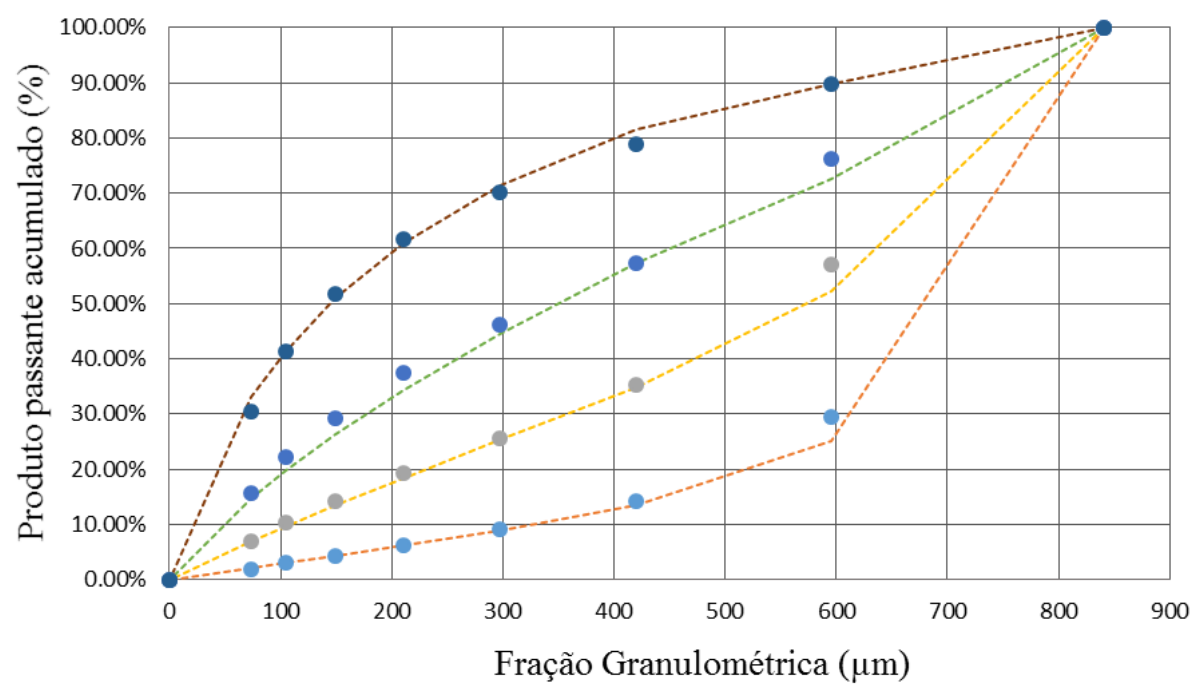

- 0.5 min medido $0.5 \mathrm{~min}$ previsto

- 1.42 min medido $1.42 \mathrm{~min}$ previsto

- 2.58 min medido

----- $2.58 \mathrm{~min}$ previsto

- 4.58 min medido

------ $4.58 \mathrm{~min}$ previsto

Fração Granulométrica $(\mu \mathrm{m})$

Figura 7: Previsão de produto final de um moinho vertical em função do tempo de moagem (linha pontilhada) e os valores medidos (pontos).

\section{CONCLUSÕES}

O Método do Balanço Populacional representou de forma satisfatória as curvas granulométricas geradas em diferentes tempos de moagem. Nesse estudo é também possível concluir que os valores da função seleção para a amostra de granito obtida em moinho vertical podem ser calculados através de um moinho de Bond utilizando a Equação 5. A similaridade das funções quebra obtidas indica que os mecanismos de cominuição são parecidos nos dois moinhos testados. Os próximos passos incluem a realização de testes com diferentes tamanhos de bolas e diferentes velocidades buscando correlações entres estas variáveis e a taxa de quebra dos moinhos estudados. 


\section{AGRADECIMENTOS}

Os autores agradecem à Hazen Research por fornecer o moinho vertical e a Capes/Ciência sem Fronteiras pelo suporte acadêmico.

\section{REFERÊNCIAS}

Austin, L., Klimpel, R. \& Luckie, P., 1984. Process Engineering of Size Reduction: Ball Milling. New York: SME.

Morrel, S., Sterns, U. \& Weller, K., 1993. The Application of Population Balance Models to Very Fine Grinding in Tower Mills. Proceeding of XIX International Mineral Processing Congress.

Morrison, R., Cleary, P. \& Sinnott, M., 2009. Using DEM to Compare the Energy Efficiency of Pilot Scale Ball and Tower Mills. Minerals Engineering, pp. 665-672.

Schonert, K., 1990. Aspects of Very Fine Grinding. Littleton: SME.

Stief, D., Lawruk, W. \& Wilson, L., 1987. Tower Mill and its Application to Fine Grinding. Minerals and Metallurgy Processing, Volume 4, pp. 45-50.

Wills, B. A. \& Napier-Munn, T., 2006. Mineral Processing Technology - An Introduction to the Practical Aspects of Ore Treatment and Mineral Recovery. Burlington: Elsevier. 